\title{
Lohnt sich die strengere Blutdruckeinstellung doch?
}

Fragestellung: Wie hoch ist das Risiko für Typ-2-Diabetiker mit einem erhöhtem systolischen und diastolischen Blutdruck eine KHK, einen Schlaganfall oder eine kardiovaskuläre Erkrankungen zu bekommen?

Hintergrund: Durch ACCORD und andere Studien ist die Diskussion um den Zielblutdruck bei Hypertoniepatienten mit Diabetes mellitus seit einiger Zeit neu entbrannt. Nun befasste sich eine weitere Studie mit dem Zielblutdruck für dieses spezielle Patientenkollektiv.

Patienten und Methodik: Die vorliegende Arbeit hat an 35041 Patienten mit Hypertonie und Typ-2-Diabetes mellitus unter antihypertensiver Behandlung und an 18512 unbehandelten $\mathrm{Pa}$ tienten im Alter zwischen 30 und 75 Jahren ohne vorbekannte Herzinsuffizienz die kardiovaskulären Ereignisse innerhalb von 6 Jahren beobachtet. Diese Beobachtungsstudie fand im Rahmen des Nationalen Schwedischen Diabetesregisters statt.

Ergebnisse: Für einen systolischen Blutdruck von $140 \mathrm{mmHg}$ und höher sowie für einen diastolischen Blutdruck von $80 \mathrm{mmHg}$ und höher waren

Cederholm J et al. Blood pressure and risk of cardiovascular diseases in type 2 diabetes: further findings from the Swedish National Diabetes Register (NDR-BPII). J Hypertens 2012, DOI:10.1097/

HJH.0b013e3283577bdf die Risiken für kardiovaskuläre Ereignisse, KHK und Schlaganfall sowie Gesamtmortalität signifikant erhöht (Abb. 1). Für den Schlaganfall gab es weder für den systolischen noch für den diastolischen Blutdruck ein J-KurvenPhänomen, d.h. bei niedrige-

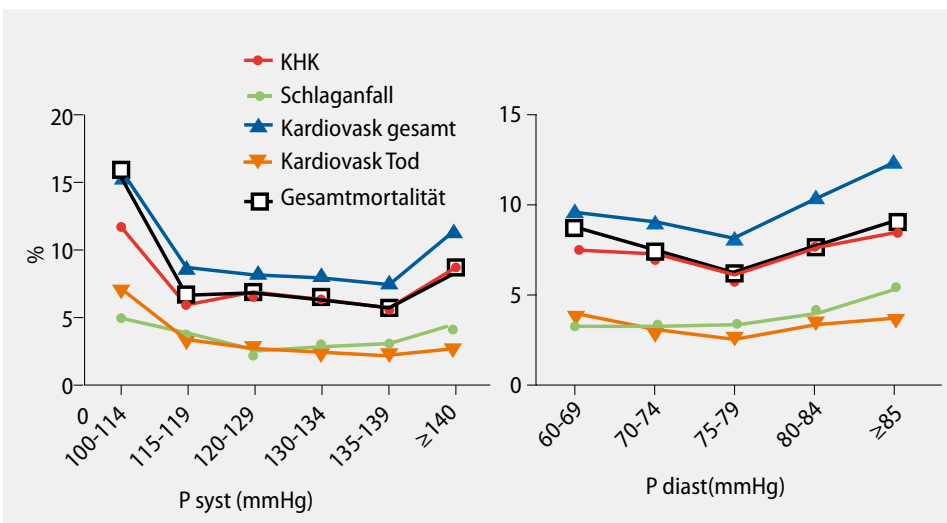

1 a und b: Häufigkeit kardiovaskulärer Ereignisse (KHK, Schlaganfall, gesamte kardiovaskulärer Ereignisse, kardiovaskulärer Todesfälle, Gesamtmortalität) in Abhängigkeit vom systolischen und diastolischen Blutdruck.

ren Blutdruckwerten bis $100 \mathrm{mmHg}$ systolisch oder $60 \mathrm{mmHg}$ diastolisch stieg die Häufigkeit von Schlaganfällen nicht an im Vergleich zu Patienten mit höherem systolischen Blutdruck.

Für die koronare Herzerkrankung und die gesamten kardiovaskulären Ereignisse fand sich im Bereich des systolischen Blutdrucks zwischen 100 und 114 mmHg eine höhere Inzidenz.

Schlussfolgerung: Typ-2-Diabetikern mit einem Blutdruck um die 130-135/75-79 mmHg haben ein geringeres kardiovaskuläres Risiko als diejenigen mit einem erhöhten Blutdruck von über 140/80 mmHg.

\section{-Kommentar von Prof. Dr. med. Walter Zidek}

\section{Die Diskussion reißt nicht ab}

Die Ergebnisse sind, wenngleich es sich nicht um eine prospektive, randomisierte Studie handelte, im Rahmen der Diskussion um den Zielblutdruck interessant. Diese Befunde weisen darauf hin, bei allen Einschränkungen durch das Studiendesign, dass hypertensive Typ-2-Diabetiker mit Blutdruckwerten deutlich unter $140 \mathrm{mmHg}$ systolisch und unter $80 \mathrm{mmHg}$ diastolisch eine bessere kardiovaskuläre Prognose haben als mit Blutdruckwerten über 140/80 $\mathrm{mmHg}$.

Auch niedrigere Blutdruckwerte waren nicht mit einer schlechteren kardiovaskulären Prognose assoziiert. Erst im Blutdruckbereich zwischen 100 und $114 \mathrm{mmHg}$ systolisch war die kardiovaskuläre Prognose schlechter. Wenngleich damit die Diskussion um den optimalen Zielblutdruck bei Hypertoniepatienten mit Diabetes nicht abgeschlossen ist, sprechen

auch diese Befunde für eine strenge Blutdruckeinstellung beim hypertensiven Typ-2-Diabetiker.

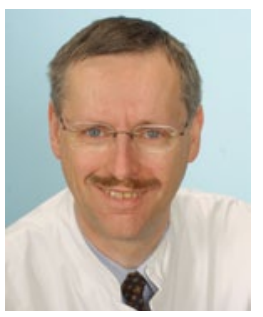

Prof. Dr. med. Walter Zidek

Medizinische Klinik IV,

Charité Berlin

Hindenburgdamm

30, 12200 Berlin 